\title{
Comprehending anaphoric presuppositions involves memory retrieval too
}

\author{
Sherry Yong Chen \& E. Matthew Husband*
}

\begin{abstract}
We investigate the memory retrieval mechanism that underlies the realtime comprehension of anaphoric presupposition triggers. Using the Drift Diffusion Model, we offer a new experimental argument for the anaphoric view of presuppositions with evidence from the memory retrieval processes associated with the trigger too. We show that the memory representation of the antecedent content that satisfies the presupposition is retrieved via a direct access mechanism, suggesting that anaphoric triggers such as too share the same processing signature of many anaphoric expressions, such as pronouns and VP ellipses.
\end{abstract}

Keywords. anaphoricity; presupposition; memory retrieval; sentence processing; experimental pragmatics

1. Introduction: Presupposition and memory. Presupposition is a class of meaning where speakers linguistically mark the information as being part of the common ground or already established in the discourse content. Presuppositions can be triggered by specific lexical items or grammatical construction; for example, the definite construction the $X$ presupposes the existence of $X$, and the appearance of too presupposes a previous action or event (Chierchia \& McConnellGinet, 1990; Beaver \& Geurts, 2012; Potts, 2015; a.o.). The theoretical literature on presupposition is vast, with the broad focus on the projection problem, the triggering problem, and how presupposition contrasts with other related aspects of meaning such as assertion and conventional implicature (Stalnaker, 1973; Heim \& Kratzer, 1998; Fox, 2008; Tonhauser et al, 2013). More recently, there has been a growing body of experimental work that investigates how presuppositions are processed in real time (Schwarz, 2014). This turn to performance-related issues raises many important questions concerning the comprehension of discourse information. Against this background, we investigate the memory retrieval mechanism that underlies the processing of presupposition triggers. How do presupposition triggers function as reliable cues during online discourse comprehension? Is presupposed content retrieved in a contentaddressable manner or by using a serial search mechanism? These issues are complex yet interesting as they have a bearing on broader architectural questions concerning the interaction of memory and the format of the representation of context during language processing.

Anaphoric dependencies have been well studied in language comprehension and have signature memory retrieval processes. The anaphoric theory of presuppositions (Kripke, 1990/2009; van der Sandt, 1992; Zeevat, 1992; Beck, 2007; a.o.) treats presuppositions analogously to anaphoric expressions such as pronouns, where "anaphoric" is taken to mean "requiring a contextually provided antecedent". For example, under this theory, the presupposition trigger too relates the asserted content and a previously established antecedent. Being anaphoric in nature, too is typically considered a "hard" trigger that is difficult to accommodate (von Fintel \& Matthewson, 2008; Abusch, 2010; Cummins, Amaral, \& Katsos, 2012). The current study focuses on the presupposition trigger too and aims to address two key

\footnotetext{
* We thank Brian Dillon, Alex Göbel, Ellen Lau, and Colin Phillips for many fruitful discussions. Thanks also to the audience at the $23^{\text {rd }}$ Architectures and Mechanisms for Language Processing Conference and the $92^{\text {nd }}$ LSA Annual Meeting for their helpful comments. Authors: Sherry Yong Chen, MIT (sychen@mit.edu); E. Matthew Husband, University of Oxford (matthew.husband@ling-phil.ox.ac.uk).
} 
questions: (1) Do anaphoric presupposition triggers initiate memory retrieval, just like other anaphoric expressions such as pronouns (Foraker \& McElree, 2011) and elliptical constructions (Martin \& McElree, 2008, 2011)? (2) If so, is this retrieval process content-addressable or search-based?

These questions are motivated by mounting evidence that presuppositions are rapidly accessed during real-time processing. Triggers such as English again and its German equivalent wieder have been reported to be rapidly sensitive to presupposition violation as revealed by reading time measures (Tiemann et al, 2011; Schwarz \& Tiemann, 2012). In addition, Romoli et al (2014) showed that the presupposition of also is used incrementally during online comprehension to predict upcoming linguistic content, which furthermore supports a rapid retrieval process of previously presupposed content in memory. The fact that retrieval processes occurs so rapidly is consistent with the hypothesis that parsers have direct access to presupposed content. On the other hand, Kim (2014) reported a locality bias associated with also in a visual world eye-tracking experiment. Participants preferred satisfying a presupposition using material that was linearly and hierarchically closer to the trigger rather than more distant in the discourse. Such locality biases in processing potentially point toward a search-based retrieval mechanism that grants local targets a temporal advantage, contrary to a straightforward direct access mechanism suggested by Romoli et al (2014).

Resolving this tension requires us to move beyond simple reading time measures and tap into the time course of memory retrieval during the processing of presupposition. In short, most existing psycholinguistic studies on presupposition have been looking into processing costs associated with presupposition violation, but exactly how a presupposition is retrieved from memory remains poorly understood.

2. Memory retrieval processes. Establishing a linguistic dependency in real-time is often thought to require the use of memory storage and retrieval as the constituent parts of the dependency are displaced from one another over sometimes unbounded length. There are currently two main hypotheses about memory retrieval on the market (McElree et al. 2003). One proposes that our mental representations of linguistic material are retrieved in a cue-based, direct-access manner. These direct access models assume that linguistic cues are used to access all potentially relevant stored representations in parallel. The main alternative proposes that memory representations are retrieved through a serial search process that retrieves potentially relevant representations one at a time. In these models, any intermediate (but ultimately irrelevant) material is initially retrieved before finding the target representation in memory. Both mechanisms predict the locality bias reported in Kim (2014). Under a cue-based direct-access mechanism, general memory decay processes cause the memory trace of distant content to be of relatively poorer quality compared to that of local content, perhaps making it a poorer candidate compared to the more recent local candidate, although both memory traces are directly retrieved in a content-addressable manner regardless of their quality. This predicts that increased dependency length affects only the final accuracy rate of retrieval but not retrieval speed. Under a serial search mechanism, increased dependency length slows down retrieval itself because memory traces are searched in a structured way, arguably one at a time. ${ }^{1}$ In addition to the

\footnotetext{
${ }^{1}$ Importantly, serial search posits that there is a relevant domain that is searched one at a time. For instance, one could propose that all of the content of a domain, say, a clause, is retrieved in parallel, but that search must still serially retrieve clause-by-clause. Concerning the architecture of retrieval for the presupposition of too, the relevant domain may be restricted to a proposition and organized in a discourse structure that is analogous to syntactic structures.
} 
degraded status of distant content driven by general memory decay processes, this hypothesis predicts that closer representations will have a temporal advantage during memory search as it is retrieved first, such that adding more distance between the presupposed content and the trigger should lead to a slowdown of the retrieval speed of more distance antecedents.

Importantly, simple experimental techniques cannot address which retrieval mechanism is at play since these techniques confound retrieval speed with accuracy. One family of methodological approaches that can disentangle speed from accuracy is the speed-accuracy trade-off design. These designs have provided a critical empirical cornerstone in our understanding of the architecture of memory retrieval in sentence processing. Much of the evidence in this domain comes from Multiple Response Speed-Accuracy Tradeoff (MR-SAT), a methodology that disassociates memory access from retrieval speed and availability, and has been key in arguing that sentence processing is mainly subserved by direct access retrieval rather than a serial search mechanism (McElree, 2000; McElree et al, 2003; cf. Dillion, et al., 2014). In this method, participants are intensively trained to rapidly respond to a series of cues with a response. This data can then be used to construct a profile of the memory retrieval process which can be characterized by three parameters: the asymptotes which reflects participant's ultimate accuracy on the task, the intercept which reflects the time when participant's accuracy departs from chance, and the rate which captures the speed at which accuracy grows from the intercept to the asymptote. Differences in either the intercept or the rate parameter provide evidence for an underlying difference in the speed of processing separate from processing accuracy. The SAT method, however, is time consuming and resource intensive, leading to fewer opportunities to replicate previous results and extend our understanding to new phenomena.

In the present study, we employed an alternative methodology, Drift Diffusion Modelling (DDM), which has been used to jointly analyse the reaction time and accuracy data produced in standard two alternative forced choice experimental tasks. This technique is motivated by the need to tease apart the effects of accuracy and speed that underlie the processing costs observed in simple reading time measures, allowing for direct modeling of the time course of retrieval. DDM jointly models accuracy and response time distributions with parameters that reflect

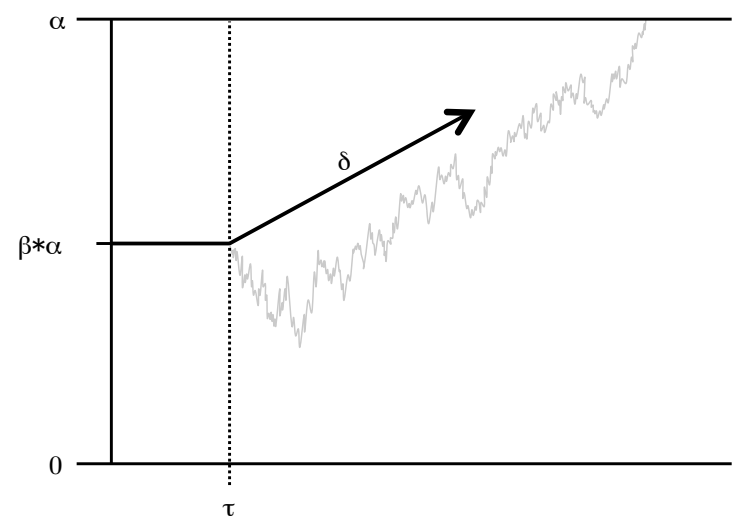

Figure 1. An illustration of the drift diffusion model. A stimulus is encountered and encoded in $\tau$ time. Afterwards, evidence for deciding between two forced choice alternatives separated by $\alpha$ begins to accumulate as a random walk, starting initially as $\alpha * \beta$ and rising by an average increment $\delta$. The process terminates when the accumulated evidence reaches either 0 or $\alpha$. 

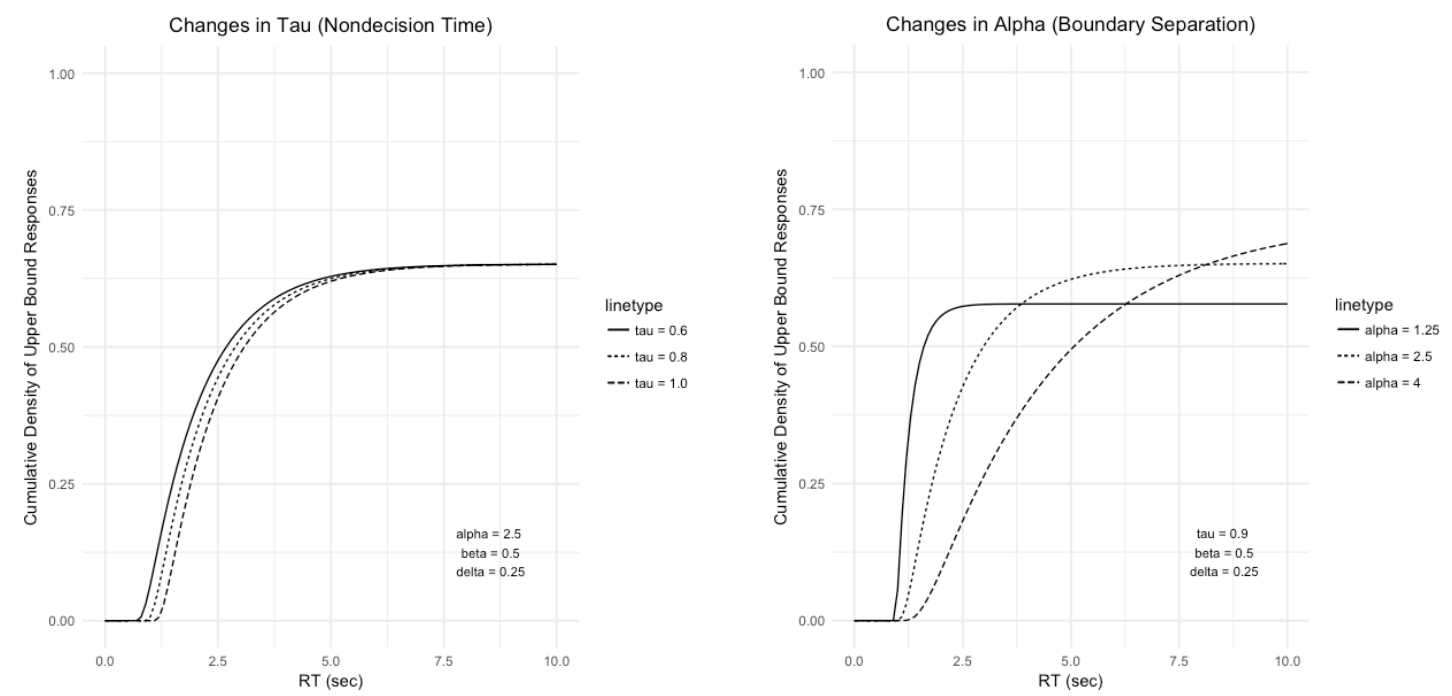

A

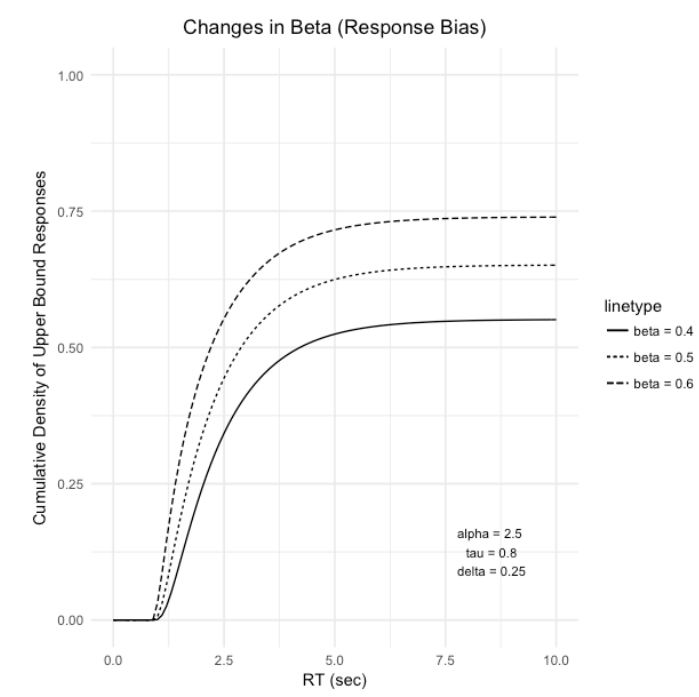

$\mathrm{C}$

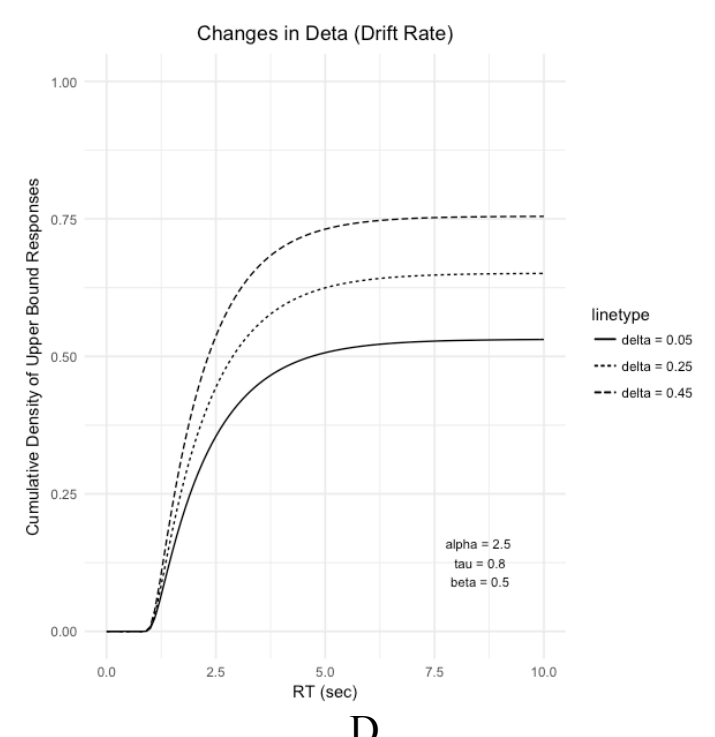

Figure 2. Hypothetical cumulative density curves for drift diffusion models that differ by only nondecision time $\tau(\mathrm{A})$, boundary separation $\alpha(\mathrm{B})$, response bias $\beta(\mathrm{C})$, or drift rate $\delta(\mathrm{D})$.

distinct underlying memory retrieval processes (Ratcliff 1978; Ratcliff, et al., 2016; McElree \& Dosher 1989), and from these measurements, a retrieval function is estimated that relates accuracy to elapsed processing time. The end product of this modelling process is a best-fitting model for the data, which consists of four crucial parameters: $\tau$, nondecision time, the encoding and motor response time; $\alpha$, boundary separation, the distance between the two possible forced choice alternative responses (with larger numbers indicating higher accuracy); $\beta$, response bias, the bias to respond to one of the forced-choice alternatives over the other (with 0.5 being no bias); and $\delta$, drift rate, the tendency of the diffusion process to drift towards one response alternative other the other (with positive values drifting upwards). A visualization of the drift diffusion process is shown in Figure 1 and the effects of each of these parameters on the model 
are illustrated in Figure 2. As we can see, the drift rate reflects asymptotic accuracy similar to the SAT asymptote, and nondecision time reflects the time when participant's accuracy departs from chance and begins to grow (assuming that motor response timing is constant within our task), similar to the SAT intercept. The other two parameters, boundary separation and response bias are related to both the rate and the asymptotic behavior of accuracy.

\section{Experiment.}

3.1. PARTICIPANTS. Sixty-four English-speaking participants were recruited via Amazon Mechanical Turk. All participants provided their written informed consent to participate in the experiment and received monetary compensation ${ }^{2}$.

3.2. DESIGN \& PROCEDURE. We examined the memory retrieval processes of too in an experimenter-paced phrase-by-phrase sentence reading task with RSVP presentation (SOA: 500 msec, ISI: $200 \mathrm{msec}$ ), followed by an end-of-sentence acceptability judgment with binary choices $(\mathrm{N}=64)$. Distance between too and the presupposed content was manipulated as Near or Far, and the Context was Same or Different, satisfying or violating the presuppositions respectively:

\begin{tabular}{cll}
\multicolumn{2}{c}{ Same } & \multicolumn{1}{c}{ Different } \\
\hline Near & $\begin{array}{l}\text { If the editor resigned, then the } \\
\text { critics resigned too. }\end{array}$ & $\begin{array}{l}* \text { If the editor plagiarized, then } \\
\text { the critics resigned too. }\end{array}$ \\
Far & $\begin{array}{l}\text { If the editor resigned, then } \\
\text { everyone from the publishing } \\
\text { house would be shocked to hear } \\
\text { that the critics resigned too. }\end{array}$ & $\begin{array}{l}* \text { If the editor plagiarized, then } \\
\text { everyone from the publishing } \\
\text { house would be shocked to hear } \\
\text { that the critics resigned too. }\end{array}$ \\
\hline
\end{tabular}

Table 1: Sample experimental material

Note that the presuppositions of too was embedded within the conditional sentence frame "if ... then ...". In these cases, the presuppositions of too are not suspended or cancelled, but are said to be "linked up or bound to a previously established antecedent" just like pronouns (van der Sandt, 1992). This design is crucial to our experiment: if the presuppositions were unembedded, too would be obligatory in the discourse (Heim, 1991; Bade, 2016), which might lead the comprehenders to develop strategic processing.

The experiment hosted on IbexFarm (Drummond, 2016). Participants were instructed to read the sentences, and then use the keyboard to indicate whether the sentence as either "bad" or "good" as quickly as possible. There were seven practical trials, followed by a total of 64 critical items and 68 fillers. The four conditions were distributed in a Latin Square design, and participants were randomly assigned to one of the four lists.

3.3. RESUlTS. Statistical analysis was carried out using linear mixed effect model in R (R Core Team, 2016). Two subjects were removed for falling two standard deviations below the mean accuracy rate. Two additional subjects were removed for falling two standard deviations outside the mean reaction time (RT) rate. Using model criticism (Baayen, 2008), 1.80\% of the total data was excluded from further analysis for falling 2.5 standard deviations outside of either the mean accuracy rates or the mean RTs.

${ }^{2}$ All methods were approved by Social Sciences \& Humanities Inter-Divisional Research Ethics Committee at the University of Oxford. 
The mean accuracy data by condition is summarized in Table 2 (parentheses represent standard error by participants). Overall, participants were more accurate at resolving the presupposition dependency in the Near condition: linear mixed effect model revealed that there was a main effect of Distance $(t=4.769, \mathrm{p}<.001)$ and Context type $(\mathrm{t}=3.604, \mathrm{p}<.001)$. Their interaction was non-significant $(\mathrm{t}=0.671, \mathrm{p}=.502)$.

\begin{tabular}{ccc} 
& Same & Different \\
\hline Near & $0.85(0.15)$ & $0.64(0.13)$ \\
Far & $0.76(0.14)$ & $0.56(0.12)$ \\
\hline
\end{tabular}

Table 2: Accuracy measures

The average RT data by condition is summarized in Table 3 (parentheses represent standard error by participants). An analysis of the RT data revealed that there was a main effect of Context type $(\mathrm{t}=-2.755, \mathrm{p}=.007)$, and a marginally significant interaction between Distance and Context type $(\mathrm{t}=1.799, \mathrm{p}=.079)$; however, planned comparison revealed no effects of Distance within the Context types.

\begin{tabular}{ccc} 
& Same & Different \\
\hline Near & $1373(0.08)$ & $1594(0.08)$ \\
Far & $1429(0.08)$ & $1515(0.08)$ \\
\hline
\end{tabular}

Table 3: Average reaction time measures in msec.

3.4. DDM ANALYSIS. Results from accuracy and reaction time measures suggest that Distance has an effect on overall accuracy, but not the speed of retrieval. To further model our data, we used the RWeiner package in R to fit a Weiner drift diffusion model for each condition for our participants (Wabersich \& Vandekerchkhove, 2014). Responses were coded for accuracy, permitting us to hold $\beta$ constant at $0.5 .^{3}$ The by-participant parameter fits were the submitted for analysis using mixed-effects modeling with maximal random intercepts and slopes by participant (parameter values that did not converge were excluded from analysis). The average model parameters for each condition are summarized in Table 4, and $t$-values for model estimates of effects on DDM parameters are in Table 5. We found a significant effect of Context on both nondecision time $\tau$ and drift rate $\delta$ (Distance: $t_{\alpha}=0.863, p_{\alpha}=0.393 ; t_{\delta}=-1.725, p_{\delta}=.090$; Context: $\left.t_{\alpha}=0.863, p_{\alpha}=0.393 ; t_{\delta}=4.261, p_{\delta}<.001\right)$, but no significant effect of Distance was found (Distance: $t_{\tau}=-1.691, p_{\tau}=.094$; Context: : $t_{\tau}=-2.100, p_{\tau}=.040$ ). The lack of a distance effect, especially on nondecision time, suggests that memory retrieval for the content of an anaphoric presupposition is accomplished by direct access.

\begin{tabular}{ccccc} 
Context & Distance & $\tau$ & $\alpha$ & $\delta$ \\
\hline Same & Near & 0.751 & 2.35 & 0.973 \\
& Far & 0.741 & 2.47 & 0.085 \\
Different & Near & 0.853 & 2.26 & 0.397 \\
& Far & 0.792 & 2.35 & 0.007 \\
\hline
\end{tabular}

Table 4: Drift diffusion model parameters

\footnotetext{
${ }^{3}$ Models that allowed $\beta$ to also vary freely produced similar results and we do not discuss them further in this paper.
} 


\begin{tabular}{cccc} 
& $\tau$ & $\alpha$ & $\delta$ \\
\hline Distance & 1.691 & 0.863 & -1.725 \\
Context & $2.100^{*}$ & 0.821 & $4.261^{* * *}$ \\
Interaction & 0.941 & -0.051 & -0.310
\end{tabular}

Table 5: $t$-values for model estimates of effects on DDM parameters

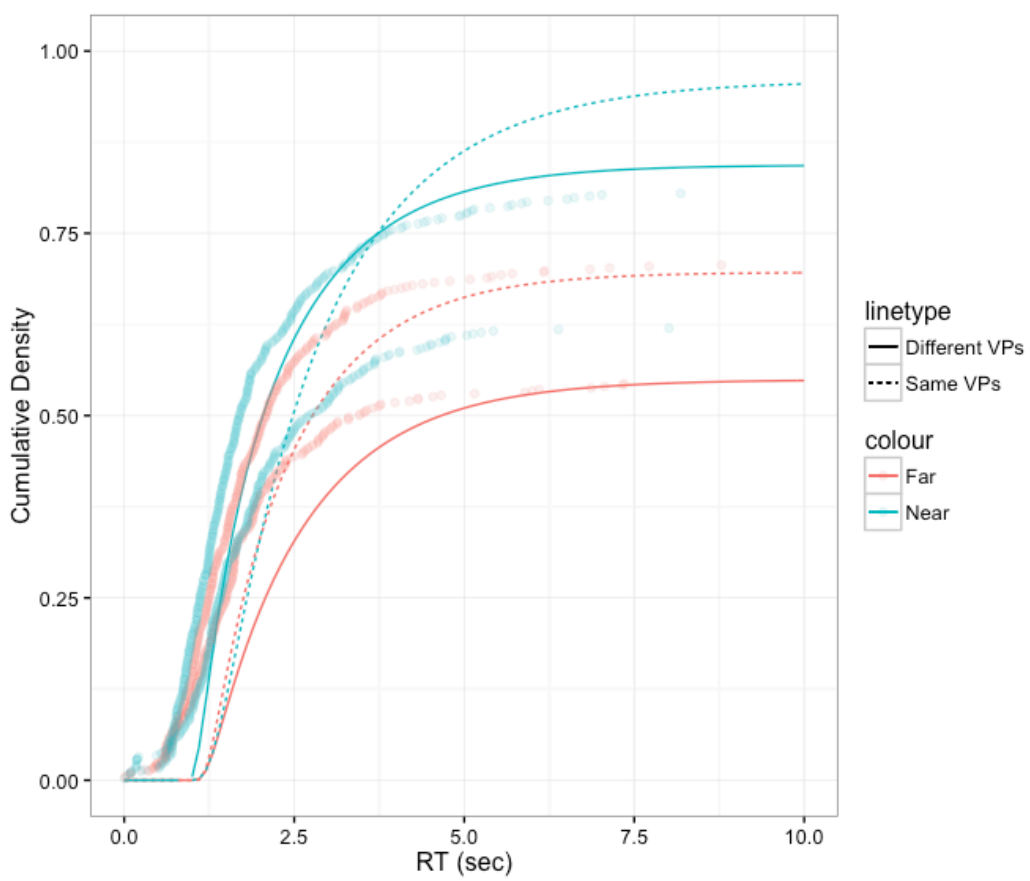

Figure 3. DDM estimates over empirical cumulative density

4. Discussion Our results are consistent with an anaphoric processing view of presuppositions. Specifically, they demonstrate that the resolution of anaphoric presuppositions involves a memory retrieval process, similar to many other anaphoric dependencies. Furthermore, our results suggest that the antecedent content of the presupposition of too is retrieved via direct access using a content-addressable mechanism. Comprehenders are able to directly access all potential candidates for presupposed content that has been established in the discourse directly, instead of searching incrementally through potential but ultimately irrelevant intermediate possibilities before finding the desired candidate in memory.

The mechanism we envision goes as follows. In the case of processing the presupposition of too, upon encountering the first clause, the comprehender encodes the representation of an event in their memory. Upon seeing the last clause and the trigger too, a memory retrieval process is initiated, during which the representation of the first event is retrieved and checked against the second event to see if presupposition has been satisfied or violated, potentially alongside any other similarly matching clausal representations. We further propose that this memory retrieval process is mandatory; if the retrieval of an antecedent that satisfies the presupposition of too fails, then comprehenders are required to decide if accommodation is needed/possible and then ultimately accept or reject the presupposition. Crucially, a serial search process would be burdensome for the comprehender as it immediately raises the question of when comprehenders would know that memory retrieval has ultimately failed in the case of presupposition violation. 
In contrast, a direct-access process allows memory retrieval to be accomplished in a single step, allowing comprehenders to both rapidly incorporate presupposed content or trigger accommodation/rejection in the case of retrieval failure. These findings open up many interesting questions regarding the comprehension of presuppositions in discourse. We now discuss some of them in the following sections in the interest of developing future work.

4.1. DiSCOURSE CUES. Given that a direct access retrieval mechanism is typically cue-based, an question immediately arises from our finding: What "cues", or combinations of "cues" are being exploited to retrieve the memory representations of presupposed content?

The answer to this question seems much more straightforward in the case of pronouns: morpho-syntactic cues such as gender and number can serve as cues in the memory representation of an entity. In the case of elliptical constructions, Martin and McElree (2011) suggest that the cues provided by the verb at the dependency site or grammatical constraints on sluicing may function as cues for direct-access retrieval. In the case of too, multiple cues could be responsible for retrieval, including syntactic cues like [VP] or [IP], or more semantic/discourse related cues like [event] or [discourse event]. It is also possible that the content of the presupposition plays a role in retrieval. Determining which of these cues are used during retrieval of a presupposition will require further experimental research.

One limitation that arises in the current design is that, in order to make sure the presupposition of too was satisfied, we used same verb as the antecedent and at the site of dependency (i.e. If the editor resigned, then the critics resigned too.). This raises the question of what exactly the comprehender is searching for in their memory: is it indeed the memory representation of a previous event, or is it simply the representation of the same verb form? This issue will be addressed in future work by using synonymous verbs to satisfy the presupposition, such that the comprehender cannot successfully retrieved an antecedent if only the form but not the meaning is accessed.

4.2. ACCOMMODATION \& CONTEXT UDPATE. In the case of presupposition violation, comprehenders may initiate an attempt to accommodate the presupposition. The fact that comprehenders know when accommodation is needed follows from the idea that the presupposed content is accessible from memory: some attempt at the retrieval of the memory representation of presuppositions needs to be made to determine whether accommodation is needed. But how is the context updated in the case of presupposition violation?

Immediately related to this question is the mounting theoretical and experimental evidence that triggers may be "hard" or "soft" depending of the possibility of accommodation (Abusch, 2010; cf. Jayez et al, 2014) and the extent to which accommodation is possible (Beaver \& Zeevat, 2012), which brings us to the third research question that explores whether different triggers may exhibit different online processing behaviors. Anaphoric triggers such as pronouns and also/too are found to be hard to accommodate, whereas factives and aspectual predicates are found to accommodate easily (Schwarz, 2014). This distinction between hard and soft triggers raises the questions of how context update may work when considered in light of the memory retrieval process. Accommodation of a presupposition can be thought of as a recovery strategy that is initiated when memory retrieval fails. It could be that the distinction between hard and soft triggers is directly related to memory retrieval mechanisms. For instance, a direct-access, cue-based retrieval mechanism may be best suited for hard triggers during the processing of presuppositions since these require exhaustive search of context which would be burdensome for a search-based mechanism. However, the situation is less clear for soft triggers. The fact that 
they are easier to accommodate and perhaps even non-anaphoric in nature suggests that the consequences of triggering an accommodation process due to an accidental memory retrieval failure are less severe. This suggests that the constraints on possible memory mechanisms may be looser for soft triggers than for hard ones, suggesting several empirically testable possibilities.

4.3. DiSCOURSE STRUCTURE. In addition to the anaphoric nature of many presuppositions which guided consideration of the memory mechanisms underlying presupposition processing, presuppositions play a further role in how a sentence relates to broader discourse context. While our study focused on distance effects of presupposition satisfaction within a sentence, in daily speech, the occurrence of the trigger too can often be sentences away from the antecedent. In these cases, one may wonder how distance should be represented when the antecedent that satisfies the presupposition, and whether the same kind of retrieval mechanism underlies this process. To this end, a theory of the hierarchy of discourse content such as Question Under Discussion (QUD) (Roberts, 2012) offers a testable hypothesis. The QUD theory suggests that information in discourse is actively structured and used to guide the anticipation of upcoming linguistic content, with evidence from both offline and online processing (Roberts, 2012; Clifton \& Frazier, 2012). A natural extension of this idea is that discourse structure may affect how comprehenders search in memory, although details of this influence remain rather unclear at this point, and we will leave this for our future work.

4.4. EXTENDING DDM. In this study, we have shown that DDM can be used to model speedaccuracy tradeoffs during the memory retrieval of anaphoric presuppositions. We have also obtained preliminary results from a replication of Martin \& McElree's (2011) which confirmed their findings for direct access of antecedents in sluicing constructions. Specifically, a difference in $\tau$ can be used to infer whether a serial search or a direct access retrieval process is at play. Modeling memory retrieval processes with DDM provides convergent evidence to Multiple Response SAT, and has advantages in requiring fewer response time measurements to recover meaningful parameters, giving us more opportunities to extend our understanding to new phenomena.

The use of DDM may also addresses a more general concern regarding the interpretation of a direct access process: since the finding of a direct access process relies on the null effect of Distance, one may wonder if it could be due to insufficient statistical power. While this particular interpretation of memory retrieval processes is not tied up to a particular methodology, DDM, being less time consuming and resource intensive, can allow more opportunities to replicate previous results, which may help address the concern for insufficient power when interpreting null effects.

5. Summary In line with the anaphoric view of presupposition, the processing of presupposition dependency does initiate a memory retrieval process. Crucially, we show that the representation of the presupposed content is elicited directly, by virtue of their content, not by a search guided by structural position information.

That the retrieval of the presupposed content of too is via a direct access mechanism fits well with evidence for other types of linguistic dependencies, such as pronoun resolution (Foraker \& McElree, 2007), VP ellipses (Martin \& McElree, 2008), and sluicing constructions (Martin \& McElree, 2011). These findings contribute to a growing body of empirical evidence suggesting that the memory representations of discourse dependencies formed during language comprehension are content-addressable and retrieved with a direct-access process. We hope that 
future work investigating what cues are available for presupposition dependency will shed more light on the nature of cues and the overall memory architecture of language comprehension.

\section{References}

Abusch, D. 2010. Presupposition triggering from alternatives. Journal of Semantics, 27(1). 3780. https://doi.org/10.1093/jos/ffp009

Baayen, R. H. (2008). Analyzing linguistic data: A practical introduction to statistics using $R$. Cambridge University Press.

Bade, N. (2016). Obligatory presupposition triggers in discourse-empirical foundations of the theories maximize presupposition and obligatory implicatures. Tübingen: Universität Tübingen dissertation.

Beaver, D. I., \& Geurts, B. 2012. Presupposition. In E. N. Zalta (ed.), The Stanford Encyclopedia of Philosophy. Palo Alto, CA: CSLI Publications.

Beaver, D. I., \& Zeevat, H. 2012. Accommodation. In G. Ramchand \& C. Reiss (eds.), Oxford handbook of linguistic interfaces. 502-538. Oxford University Press.

Beck, S. 2007. Quantifier dependent readings of anaphoric presuppositions. In U. Sauerland \& P. Stateva (eds.), Presupposition and implicature in compositional semantics. 12-33. Palgrave Macmillan.

Chierchia, G., \& McConnell-Ginet, S. 2000. Meaning and grammar: An introduction to semantics. MIT press.

Clifton, C., \& Frazier, L. 2012. Discourse integration guided by the 'question under discussion'. Cognitive Psychology, 65(2). 352-379. https://doi.org/10.1016/j.cogpsych.2012.04.001

Cummins, C., Amaral, P., \& Katsos, N. 2012. Experimental investigations of the typology of presupposition triggers. Humana Mente, 23, 1-16.

Dillon, B. 2014. Syntactic memory in the comprehension of reflexive dependencies: an overview. Language and Linguistics Compass, 8(5). 171-187. http://dx.doi.org/10.1111/lnc3.12075

von Fintel, K. \& Matthewson, L. 2008. Universals in semantics. The Linguistic Review, 25(1-2), 139-201.

Drummond, A. 2016. IbexFarm (Version 0.3.9). Retrieved from http://spellout.net/ibexfarm/

Foraker, S., \& McElree, B. 2011. Comprehension of Linguistic Dependencies: Speed-Accuracy Tradeoff Evidence for Direct-Access Retrieval From Memory. Language and Linguistics Compass, 5(11). 764-783. http://dx.doi.org/10.1111/j.1749-818X.2011.00313.x

Fox, D. 2008. Two short notes on Schlenker's theory of presupposition projection. Theoretical Linguistics, 34(3). 237-252. https://doi.org/10.1515/THLI.2008.016

Heim, I., \& Kratzer, A. 1998. Semantics in generative grammar (Vol. 13). Oxford: Blackwell.

Jayez, J., Mongelli, V., Reboul, A., \& Van Der Henst, J. B. 2014. Weak and strong triggers. In F. Schwarz (ed.), Experimental perspectives on presuppositions. 173-193. Springer International Publishing.

Kim, C. S. 2014. Presupposition satisfaction, locality and discourse constituency. In F. Schwarz (ed.), Experimental Perspectives on Presuppositions. 109-134. Springer International Publishing.

Kripke, S. A. 2009. Presupposition and anaphora: Remarks on the formulation of the projection problem. Linguistic Inquiry, 40(3). 367-386. https://doi.org/10.1162/ling.2009.40.3.367 
Martin, A. E., \& McElree, B. 2008. A content-addressable pointer mechanism underlies comprehension of verb-phrase ellipsis. Journal of Memory and Language, 58(3). 879-906. https://doi.org/10.1016/j.jml.2007.06.010

Martin, A. E., \& McElree, B. 2011. Direct-access retrieval during sentence comprehension: evidence from sluicing. Journal of memory and language, 64(4). 327-343. https://doi.org/10.1016/j.jml.2010.12.006

McElree, B. (2000). Sentence comprehension is mediated by content-addressable memory structures. Journal of Psycholinguistic Research, 29(2), 111-123. https://doi.org/10.1023/A:1005184709695

McElree, B., Foraker, S., \& Dyer, L. 2003. Memory structures that subserve sentence comprehension. Journal of Memory and Language, 48(1). 67-91. https://doi.org/10.1016/S0749-596X(02)00515-6

Potts, C. 2015. Presupposition and Implicature. In S. Lappin \& C. Fox (eds.), The handbook of contemporary semantic theory. 168-202. Wiley-Blackwell.

R Core Team. 2016. R: a language and environment for statistical computing. R Foundation for Statistical Computing. Vienna, Austria. Retrieved from http://www.R-project.org/

Roberts, C. 2012. Information structure in discourse: Towards an integrated formal theory of pragmatics. Semantics and Pragmatics, 5(6). 1-69. https://doi.org/10.3765/sp.5.6 Romoli,

J., Khan, M., Sudo, Y., \& Snedeker, J. 2014. Resolving temporary referential ambiguity using presupposed content. In F. Schwarz (ed.), Experimental perspectives on presuppositions. 67-87. Springer International Publishing.

van der Sandt, R. A. 1992. Presupposition projection as anaphora resolution. Journal of Semantics, 9(4). 333-377. https://doi.org/10.1093/jos/9.4.333

Schwarz, F. (Ed.). 2014. Experimental perspectives on presuppositions (Vol. 45). Springer. https://doi.org/10.1007/978-3-319-07980-6

Schwarz, F., \& Tiemann, S. 2012. Presupposition Processing - The Case of German wieder. In M. Aloni, V. Kimmelman, F. Roelofsen, G. W. Sassoon, K. Schulz, \& M. Westera (Eds.), Logic, Language and Meaning. 200-209. Springer Berlin Heidelberg.

Stalnaker, R. C. 1973. Presuppositions. Journal of Philosophical Logic, 2. 447-457

Tiemann, S., Schmid, M., Bade, N., Rolke, B., Hertrich, I., Ackermann, H., Knapp, J. \& Beck, S. 2011. Psycholinguistic evidence for presuppositions: On-line and off-line data. Sinn \& Bedeutung, 15. 581-597.

Tonhauser, J., Beaver, D., Roberts, C., \& Simons, M. 2013. Toward a taxonomy of projective content. Language, 89(1). 66-109. https://doi.org/10.1353/lan.2013.0001

Wabersich, D., \& Vandekerckhove, J. (2014). The RWiener Package: an R Package Providing Distribution Functions for the Wiener Diffusion Model. $R$ Journal, 6(1).

Zeevat, H. (1992). Presupposition and accommodation in update semantics. Journal of Semantics, 9(4), 379-412. https://doi.org/10.1093/jos/9.4.379 